\title{
XMM-Newton observations of SS 433 I. EPIC spectral analysis
}

\author{
W. Brinkmann ${ }^{1}$, T. Kotani² ${ }^{2}$ and N. Kawai ${ }^{2}$ \\ 1 Max-Planck-Institut für extraterrestrische Physik, Giessenbachstrasse, 85740 Garching, Germany \\ e-mail: wpb@mpe.mpg.de \\ 2 Tokyo Institute of Technology, 2-12-1 O-okayama, Meguro-ku, Tokyo 152 - 8551, Japan
}

Received 2 August 2004/ Accepted 30 September 2004

\begin{abstract}
SS433 was observed with XMM-Newton in four consecutive orbits at different binary phases at precessional phases near maximum blue-/red shift between April 2 and April 8, 2003. The light curves in the individual orbits are flat without statistically significant indications for short-term $(\gtrsim 50 \mathrm{~s})$ intrinsic variability. Orbit-to-orbit flux changes occur predominantly in the hard band; the soft flux ( $\lesssim 2 \mathrm{keV}$ ) stays nearly constant, which implies a jet length in excess of the size of the primary star. The spectral behavior is complex and the high sensitivity and wide bandpass of the XMM-Newton instruments rule out any of the simple continuum models used previously. The data are modeled in terms of thermal emission from conically out-flowing jets and require the existence of an additional emission component, most likely from Compton scattering of the emission from the base of the jet off the cold matter from the surrounding thick disc. The temperature at the base of the jets is about $T_{0} \sim 17 \pm 2 \mathrm{keV}$. The density cannot be determined from the current data alone, however, using the most recent mass estimates for the system it must be $\sim 10^{12} \mathrm{~cm}^{-3}$ to account for the observed flux during eclipse. The kinetic energy in the jet is then $\sim 5 \times 10^{39} \mathrm{erg} \mathrm{s}^{-1}$. In the out-flowing matter Nickel is highly over-abundant ( $\sim 8$ times solar), Fe shows nearly solar abundances, and $\mathrm{Si}$ and $\mathrm{S}$ are over-abundant by a factor of $\sim 2$.
\end{abstract}

Key words. stars: individual: SS 433 - X-rays: binaries - radiation mechanisms: thermal - hydrodynamics stars: binaries: general

\section{Introduction}

The Galactic binary SS 433 emits two oppositely directed jets with velocities of $v_{j} \sim 0.26 \mathrm{c}$, which precess under an angle of $\theta=19.8^{\circ}$ with a period of $P_{\mathrm{p}}=162.5$ days (Margon 1984). The kinetic energy of the jets is enormous and might be in excess of $\sim 10^{40} \mathrm{erg} \mathrm{s}^{-1}$. SS 433 is thus regarded to be one of the most prominent objects of the class of galactic Microquasars (Mirabel \& Rodriguez 1998).

In the first phase after its discovery in 1978 most of the investigations were done at optical and at radio wavelengths (see the review by Margon 1984), and much theoretical effort was made to understand the unique properties of this object. Later, the emphasis shifted to the X-ray band which allows the study of the central regions of the relativistic jets. The relatively undistinguished X-ray source with a $2-10 \mathrm{keV}$ luminosity of a few times $10^{35} \mathrm{erg} \mathrm{s}^{-1}$ had been known from early Ariel and HEAO observations (Seward et al. 1976; Rickets et al. 1981; Marshall et al. 1979), but was only later recognized to be SS 433 from Einstein observations (Grindlay et al. 1984). Doppler shifted iron lines in the spectrum of SS 433 were detected in X-ray observations with the EXOSAT satellite (Watson et al. 1986). As the centroids of the lines follow the precessional Doppler curve and the X-rays are strongly modulated with both the precessional phase and the binary motion with period $P_{\mathrm{b}}=13.08$ days (Stewart et al. 1987) they originate in the base of the jets, emitting thermal radiation at very high temperatures. Detailed numerical calculations by Brinkmann et al. (1988) supported the picture of hot outflowing gas, which, at large distances undergoes thermal instabilities, which result in blobs of cool gas which then give rise to the observed Doppler-shifted optical line flux.

In a series of observations with the Ginga satellite from 1987 to 1991, utilizing the LAC's (Large Area Counter) higher sensitivity and wider energy range (2-35 keV, Turner et al. 1989), the behavior of the source around primary optical eclipse $\Phi \sim 0$ at different precessional phases was studied (Kawai et al. 1989; Brinkmann et al. 1989, 1991; Yuan et al. 1995). The main results of these observations were the detection of a narrow iron line following the precessional motion of the jets, and a very broad line, centered at about $7 \mathrm{keV}$, which appears to be "stationary" but changes its intensity and width with binary and precessional phase. The lines are superimposed on a very hot $(k T \gtrsim 30 \mathrm{keV})$ bremsstrahlung continuum which changes as well with binary and precessional phases. By adjusting the physical parameters in hydrodynamical simulations of an out-flowing conical jet to match the measured 
X-ray emission (Brinkmann et al. 1989) the parameters at the base of the jets could be estimated. With a number density of $n_{0} \sim(1-5) \times 10^{13} \mathrm{~cm}^{-3}$ and a temperature of $T_{0} \sim 20 \mathrm{keV}$ the kinetic energy in the jet is of the order of $\sim 10^{40-42} \mathrm{erg} \mathrm{s}^{-1}$ and the length of the X-ray emitting jet is of the order of $\gtrsim 10^{12} \mathrm{~cm}$.

Deep ASCA observations (Kotani et al. 1996) for the first time allowed estimates of the jet parameters by emission line diagnostics, using mainly iron, sulfur, and silicon lines. Recent observations with the high spectral resolution of the Chandra transmission gratings presented an enormous step forward in plasma diagnostics via emission line spectroscopy. From the measured broadening of the emission lines, Marshall et al. (2002) determined the opening angle of the jets to be $1.23 \pm$ 0 .06. By modeling the jet they obtain an electron density at the base of the visible jet of $n_{\mathrm{e}} \sim 2 \times 10^{15} \mathrm{~cm}^{-3}$ and temperatures ranging from $5 \times 10^{6} \mathrm{~K}$ to $1 \times 10^{8} \mathrm{~K}$. From a later Chandra HETGS observation of SS 433 , Namiki et al. (2003) deduced initial temperatures of $19.5_{-9.5}^{+7.8} \mathrm{keV}$ and opening angles from the Si lines of $\Theta_{\mathrm{Si}}=0.8 \pm 0.2$ and from the iron lines of $\Theta_{\mathrm{Fe}}=2.1_{-0.3}^{+0.6}$. They attribute the differences to an unusually low activity state of SS 433 during the Marshall et al. observation. From another, very recent, Chandra observation Lopez et al. (2003) find broader emission lines and, correspondingly, a wider opening angle of the jets of $2^{\circ} .70 \pm 0^{\circ} .32$.

For an astrophysical system the physical parameters of the jets are extremely well determined, with all relevant quantities known to about an order of magnitude. This still implies large uncertainties in some critical parameters of the system, like the mass outflow rate from the compact object and the energy deposition rate into the surrounding supernova remnant W50 (Yamauchi et al. 1994, Brinkmann et al. 1996). However, despite considerable observational and theoretical efforts, many details of the system, its evolutionary origin, the nature of the compact object and the jet acceleration mechanism, remain poorly understood. This is partly due to the inherent complexity of the system, showing strong temporal variability and systematic changes of the emission with the two, observationally unpleasantly long, binary and precessional periods. Further, our knowledge of the jets' parameters is mainly based on comparisons of simple hydro-dynamical models with the results from X-ray observations (Brinkmann et al. 1989; Yuan et al. 1995; Kotani et al. 1996; Marshall et al. 2002; Namiki et al. 2003). In these models the jet is treated as a hydro-dynamical, purely radially out-flowing conical flow. The plasma is optically thin and in collisional ionization equilibrium. So far the deviations from more exact flow solutions could not be tested observationally - especially as the more realistic jet models (Brinkmann et al. 1999) predict only moderate differences to the emission characteristics from the simple conical jet model.

However, two aspects of the X-ray jets remain unique: the length of the jets is of the same order as the dimensions of the binary systems and thus occultation observations can be used to directly determine the size of the primary star and the orbital separation (Stewart et al. 1987; Brinkmann et al. 1989). Secondly, the jets present an ideal laboratory for our understanding of the jet phenomenon itself, and allow comparisons of numerical jet models with observational facts.
The recent availability of emission line spectroscopy promises a much more accurate determination of the physical parameters of the out-flowing plasma, however, not without some caveats. We are dealing with the superposition of at least two systems of plasmas with highly non-uniform temperatures, densities, and emission measures. The role of photo ionization in the plasmas has not yet been determined accurately and, although the two jets are commonly assumed to be "similar" they are physically distinct systems. Most of the energy is emitted in the continuum which should therefore be an indispensable tool for the determination of the plasma temperature and energy budget. XMM-Newton observations with the large sensitivity and the wide energy band of the EPIC instruments should thus provide a reliable mapping of the emission characteristics of the jets.

In the next section we will describe the observations and the basic techniques of the data analysis. We start the spectral modeling with general considerations related to the temporal variability and the complexity of the system. We then describe the spectral jet models and give details of the spectral fitting process. We first use the data from the longest observation, orbit 610 , and determine physical parameters from the high energy data. From the low-energy data we estimate the contributions of the red shifted jet to the total flux. We then use the results to study the system during different binary phases, in the observations during the first three satellite orbits (607-609). We will finally discuss the results in the framework of the canonical jet model and the prospects of determining the system's parameters via X-ray observations.

\section{The data}

SS 433 was observed in four consecutive orbits by XMM-Newton on April 2-12, 2003. The EPIC PN camera was operated in Small Window mode with a medium filter (for details of the XMM instruments see Ehle et al. 2001). The MOS1 camera was operated in Timing mode, the MOS2 camera in Full Window mode, both with a medium filter as well. The two RGS were used in the Spectroscopy High Event Rate mode; the OM was operated in Imaging mode with the GRISM2. The observational details of the EPIC instruments for the four pointings are reported in Table 2. As exposure we give the actual Live Time of the detector. As count rate we list the ratio of the total number of events on the chip divided by the exposure to indicate the work load of the detectors. This rate is not necessarily a direct measure of the source strength; for example in orbit 608 a large fraction of the recorded counts originate from strong background flares during that orbit. The last column gives the background subtracted average count rates used in the spectral fits after the appropriate screening. All PN and MOS data were reprocessed using the latest XMMSAS version 6.0, released in March 2004.

Due to the extremely unfavorable visibility conditions of SS 433 it had to be scheduled at the beginning of the individual orbits, after the satellite's egress from perigee. As the backgrounds were rather high, for only a fraction of the scheduled time could we obtain useful data. The achieved statistical quality of the data is high enough to rule out some previously used 
Table 1. Details of the observations.

\begin{tabular}{ccccccrr}
\hline \hline Orbit & $\begin{array}{c}\text { Observing date } \\
\text { UT }\end{array}$ & Instrument & Mode & Filter & $\begin{array}{c}\text { Exposure } \\
(\mathrm{ks})\end{array}$ & $\begin{array}{c}\text { Count rate } \\
\text { cts/s } \mathbf{s}^{(\dagger)}\end{array}$ & $\begin{array}{c}\text { Rate in fit } \\
\text { cts/s } / \mathrm{s}^{(\ddagger)}\end{array}$ \\
\hline 607 & April 02,2003 & PN & Small Window & Medium & 5.37 & 43.8 & 18.91 \\
& 16:19-18:27 & MOS1 & Timing & Medium & 0.14 & - & - \\
& & MOS2 & Full Window & Medium & 0.55 & - & - \\
\hline 608 & April 04, 2003 & PN & Small Window & Medium & 8.47 & 56.1 & 27.69 \\
& $13: 12-18: 01$ & MOS1 & Timing & Medium & 5.22 & 12.6 & 6.48 \\
& & MOS2 & Full Window & Medium & 5.09 & 21.6 & 4.73 \\
\hline 609 & April 06, 2003 & PN & Small Window & Medium & 4.41 & 42.4 & 29.82 \\
& 13:04-17:53 & MOS1 & Timing & Medium & 5.35 & 12.6 & 7.11 \\
& & MOS2 & Full Window & Medium & 5.77 & 16.6 & 5.04 \\
\hline 610 & April 08, 2003 & PN & Small Window & Medium & 8.48 & 46.8 & 33.77 \\
& $12: 56-17: 45$ & MOS1 & Timing & Medium & 12.54 & 13.5 & 7.85 \\
& & MOS2 & Full Window & Medium & 12.81 & 14.2 & 5.41 \\
\hline
\end{tabular}

$\dagger$ This count rate is the total number of counts on the chip, divided by the exposure.

\# Background subtracted count rates for the spectral fits.

simple models, but in many respects the actually-scheduled longer observations would have been desirable.

\subsection{Inter-calibration / light curves}

SS 433 displays a complex thermal spectrum with a multitude of emission lines, which varies considerably with the precessional and binary phases of the system (Yuan et al. 1995). During the XMM-observations the precessional phases should be in the range $0.50 \lesssim \Psi \lesssim 0.55$ according to the ephemeris of Margon \& Anderson (1989), Goranskii et al. (1998), or Eikenberry et al. (2001), i.e., near the maximal values of the Doppler shifts from the kinematic model. Note that the systematic uncertainties and the frequently occurring erratic changes of the Doppler shifts did not allow a more precise a priori prediction of the actual phases during the time of the observations. The orbital phases change from $\Phi \sim 0.02$ at the beginning of the observations on April 2, 2003 to $\Phi \sim 0.5$ at the end on April 8, 2003 (Goranskii et al. 1998), i.e. we expect the source to be eclipsed during the first observation.

We performed the temporal and spectral analysis of the $\mathrm{PN}$ and MOS data using the latest available versions of the response matrices, released in March 2004 (PN). The MOS response matrices and effective areas were created with the SAS packages rmfgen and arfgen, respectively. As a preliminary step, we created Good Time Interval (GTI) files to check for time intervals with high background which could contaminate significantly the source photons. However, due to the generally high background we had to accept data sets for the spectral analysis with higher background levels than usually recommended.

For the PN the source photons were extracted from a rectangular region (in detector RAW pixels) of $\sim 110^{\prime \prime} \times 110^{\prime \prime}$ around the source, avoiding the chip boundary, with patterns $0-4$ (singles and doubles) and with quality flag $=0$. For the MOS2 we used a circular region centered on the source with a radius of $50^{\prime \prime}$, with patterns $0-12$ and with quality flag $=0$ as well. For the MOS1 in Timing Mode we used photons from the columns $308 \leq$ RAWX $\leq 328$. In all cases the selection regions contain about $90 \%$ of the source photons, using the encircled energy function given by Ghizzardi \& Molendi (2001).

The backgrounds were determined with the same selection criteria from source free regions on the same chips.

Figure 1 shows the background subtracted PN light curves of the four orbits in the $0.2-12 \mathrm{keV}$ band, binned in $100 \mathrm{~s}$ bins. The count rates were not corrected for the $\sim 71 \%$ Live Time of the detector in SW mode. In all four observations the light curves are essentially flat with no indications of statistically significant systematic variability. Most of the apparent "scatter" in the light curves must be attributed to the very strong and flaring backgrounds during the observing windows at the early phases of the satellite orbit. Background flares reach count rates of up to more than $50 \%$ of the source count rates which leads to a substantial degradation of the observing efficiency for the following spectral analysis, as mentioned before.

The average count rates clearly increase from orbit to orbit, confirming the predictions that in orbit 607 the source was eclipsed and then increased its flux during egress out of eclipse. Unfortunately, the short exposures do not provide sufficient coverage for a modeling of the egress process. In all four light curves there are indications of a slightly decreasing count rate with time in the hard band $(\$ 10 \%$ in $5 \mathrm{ks})$. This might be related to the decreasing background activity during the observations. On the other hand in previous observations SS 433 showed complex light curves after egress out of primary eclipse and at early binary phases. From the energyresolved light curves we find that the soft flux $(\leq 2 \mathrm{keV})$ stays basically constant at the same level in the last three pointings; only during the eclipse does the soft flux decrease to a slightly lower level. The count rate differences between the individual exposures are caused by the varying hard flux $(\geq 3 \mathrm{keV})$ by up to a factor of two. 


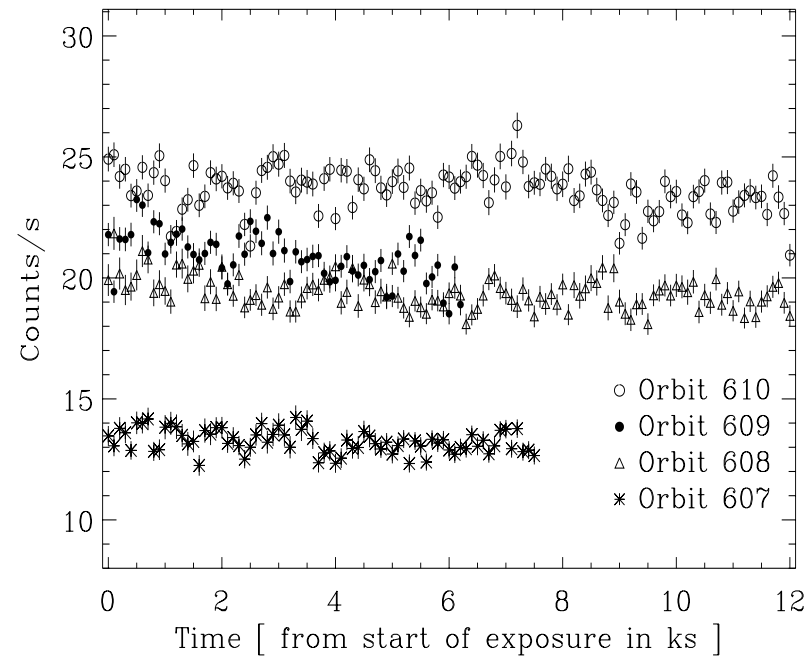

Fig. 1. PN background subtracted light curve of SS 433 during the $\mathrm{XMM}$ orbits 607-610; the time binning is $100 \mathrm{~s}$. The error bars are of the same size as the symbols.

The complex spectral behavior of the source is illustrated in Fig. 2 where we plotted the raw spectra of SS 433 during the four observations in the hard energy band. For the clarity of the plots the spectra have been shifted arbitrarily in the vertical direction. In reality, the continuum count rate in orbit 610 is about twice as high as that of orbit 607 (eclipse) whereas orbits $608 / 609$ have intermediate and very similar continuum fluxes. Whereas the (raw) count rates of the red-and blue shifted iron lines are usually comparable, in orbit 608 the blue shifted line is considerably stronger, reaching the same flux as that of orbit 610 despite the lower continuum flux.

During the observations the precessional phase increases towards larger Doppler shifts, according to the kinematic model, by $\Delta z \sim \pm 4 \times 10^{-3}$ per day. In fact, during the observation span of 6 days the emission lines seem to have "moved" by the appropriate amount, but basically in one step between orbit 608 and 609. This behavior is very likely related to the nodding motion of the disc (see Gies et al. 2002a; Hillwig et al. 2004) with the much shorter time scale of $\sim 6.3$ days.

In orbit 607 the central, hotter parts of the X-ray source are eclipsed by the primary and, correspondingly, the red and the blue shifted iron lines are of comparable strength. After egress out of eclipse (orbit 608) the blue shifted line is unusually strong, and the spectrum shows very pronounced blue shifted Fe XXVI and Nickel lines, indicative of very special viewing conditions in the source. Finally, in orbit 610 the binary phase is $\Phi \sim 0.5$, the blue shifted jet is seen basically un-obscured but the red shifted component is largely hidden by the accretion disc.

Of particular interest for a source modeling is also the spectral region around $6.5 \mathrm{keV}$ where we expect the contribution of neutral iron emission from the accretion disc or the surrounding matter (Kotani et al. 1996).

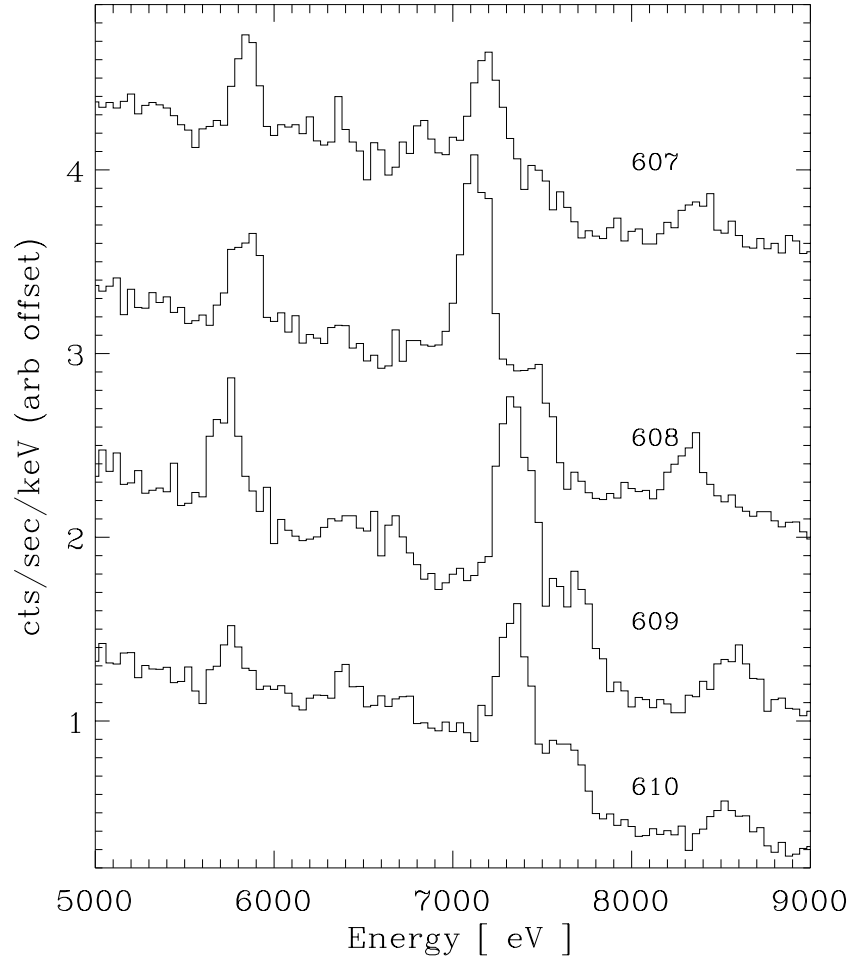

Fig. 2. PN raw spectra (binning $40 \mathrm{eV}$ ) of SS 433 during the four observations in the 5-9 keV energy range. For plotting reasons the histograms have been shifted by $3.3 \mathrm{cts} / \mathrm{s} / \mathrm{keV}$ (orbit 607 ), $1.6 \mathrm{cts} / \mathrm{s} / \mathrm{keV}$ (orbit 608), and $0.6 \mathrm{cts} / \mathrm{s} / \mathrm{keV}$ (orbit 609).

\section{Spectral modeling}

\subsection{General considerations}

For a comparison of the different detectors (and observing modes) we started the analysis with the observation of orbit 610 as it has the longest exposure and the binary phase of the system is such that we see the X-ray source un-obscured (see below and Table 2) at binary phase $\Phi \sim 0.5$.

Figure 3 shows the raw spectra from orbit 610 , selected according to the above criteria, for the PN (top) and the two MOS cameras. The complex structure is clearly visible as a multitude of emission lines superimposed on a hard continuum. SS 433 is heavily absorbed by a column density of the order of $N_{\mathrm{H}} \sim 8 \times 10^{21} \mathrm{~cm}^{-2}$ (Brinkmann et al. 1996). The exact value is not yet known, due to the earlier inadequacies of spectral modeling at lower energies and the spectral resolution of previous instruments. Unfortunately, this high absorption leaves only a relatively weak signal in the two RGS detectors, too weak for any detailed line spectroscopy.

The spectrum cannot be fitted with any simple continuum law. Excluding the line dominated region, 5.5-9 keV, from the fit, neither the earlier used thermal bremsstrahlung laws (EXOSAT, Ginga) nor a simple power law (ASCA) can describe the broad band continuum satisfactorily. For the data with lower statistics (orbit 607) a broken power law describes the broad band spectrum quite well. But in all cases the models cannot reproduce the slight curvatures inherent in the data and the large number of smaller residuals at energies where 


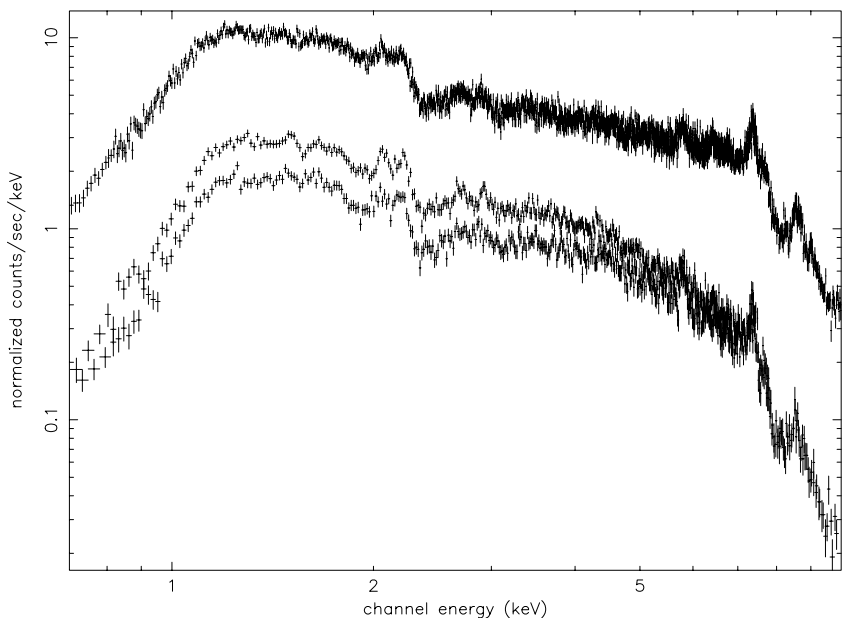

Fig. 3. Raw spectra from orbit 610; top: PN data, middle: MOS-1 data (timing mode), and bottom: MOS-2 data (full window mode)

emission structures of a thermal plasma are expected. Formally, a Comptonization model (compTT, Titarchuk 1994) provides an acceptable fit to the continuum $\left(\chi_{\text {red }}^{2}=1.48 / 884\right.$ d.o.f. $)$ with a temperature of the scattering electrons of $k T \sim 2.8 \mathrm{keV}$ and an optical depth $\tau \sim 10$. However, the residuals indicate that at higher energies the curvature of the model gets progressively too large and, further, the physical interpretation of this model remains unclear. After all, the large number of line features in the spectrum are clear indicators of a predominantly thermal origin of the spectrum. Fits with simple continuum models plus multiple Gaussian lines exceed the statistical quality of the data and the resolving power of the CCD detectors and thus make them unreliable as well.

In particular, the determination of the jet temperature from the line ratio of the Fe XXVI $\mathrm{K}_{\alpha}$ to the Fe XXV $\mathrm{K}_{\alpha}$ lines (Kotani et al. 1996; Brinkmann \& Kawai 2000) has considerable errors just from the uncertainty of the form of the underlying continuum. Fits with Gaussian lines on top of various different continuum models in the $5-10 \mathrm{keV}$ range yield temperatures at the base of the jet of $13.3 \leq k T_{0} \leq 15.9 \mathrm{keV}$; by including the errors of the fitted line parameters this range is enlarged to $12.7 \leq k T_{0} \leq 18.2 \mathrm{keV}$.

As the emission is expected to originate from the superposition of the two expanding and cooling jets, we tried to fit spectra obtained from numerical simulations of the jets (for details see Brinkmann \& Kawai 2000) to the data. In the standard "conical jet" models the emitted spectrum is mainly governed by the temperature $T_{0}$ of the gas at the base of the jet and the observed spectrum is the superposition of the blue-shifted emission from the jet pointing towards us plus a certain fraction of the emission from the red-shifted jet. As the inner parts of this "red" jet are obscured by the disc, the spectral contribution from the outer red jet is mainly expected at softer X-ray energies (for details see next section below).

It turned out that we could not model the data with any such calculated spectra; the measured spectrum in the $\sim 5-8 \mathrm{keV}$ band appears to be too hard for an underlying bremsstrahlung continuum. This is demonstrated in Fig. 4 where we fit a simple thermal spectrum with temperature $k T=40 \mathrm{keV}$ to the

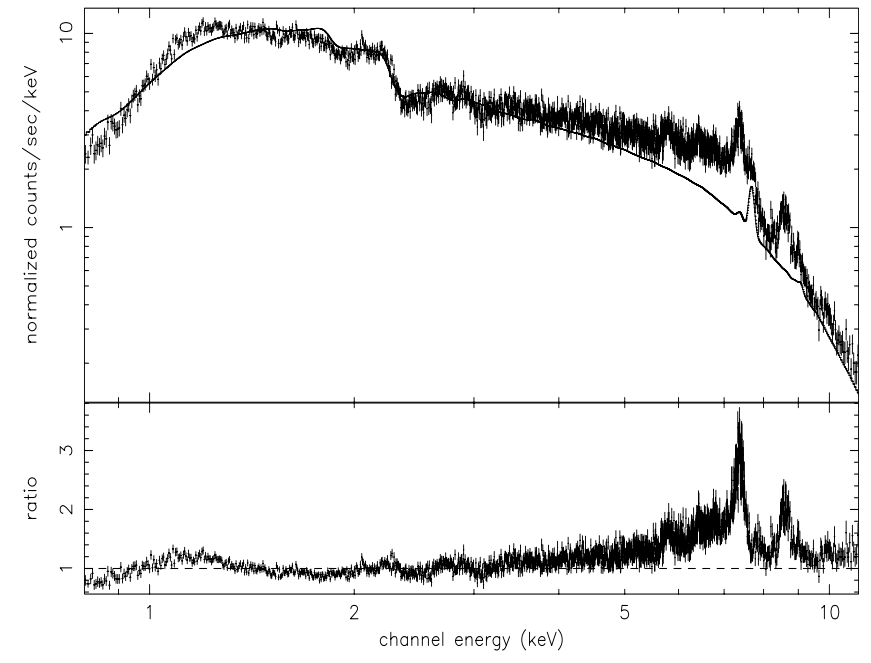

Fig. 4. Spectral fit of a single bremsstrahlung spectrum with $k T=$ $40 \mathrm{keV}$ to the PN data of orbit 610 .

data. This temperature is the upper limit of temperatures ever quoted for SS 433 (Yuan et al. 1995); the usually favored values are around $k T \sim 20 \mathrm{keV}$. Clearly, the iron line emission of this very hot plasma is inconsistent with the data and at low energies some flux is missing, but the critical difference is the surplus of observed flux in the $5-8 \mathrm{keV}$ energy range.

An obvious way to obtain a harder spectrum would be to include electron-electron bremsstrahlung into the plasma code. This radiation has a harder spectrum and becomes progressively more important at higher temperatures (Haug 1989). However, even at a plasma temperature of $40 \mathrm{keV}$ electron-electron bremsstrahlung contributes only $\sim 12.7 \%$ to the photon flux at $\sim 7 \mathrm{keV}$, insufficient to account for the deficit visible in Fig. 4.

Other components which could be physically justified result in unacceptable fits: an additional power law exceeds the data at higher energies and an additional thermal component, heavily absorbed at lower energies, predicts an absorption edge at $\sim 7 \mathrm{keV}$, not visible in the data.

The most promising and physically motivated extra spectral component would be the reflection/scattering of the jet's radiation from the accretion disc and the surrounding material. This kind of scenario has already been proposed by Brinkmann et al. (1991) from Ginga observations as explanation for the broad "stationary" iron line, not following the precessional energy shifts. Further, there are indications for a line structure in the spectra at around $6.4 \mathrm{keV}$, supporting this reflection/scattering scenario. This feature seemed to be present as well in the ASCA data (Kotani et al. 1996).

Due to the geometrical complexity of the scattering region it is currently not possible to get a reliable spectral form for this component. A single broad emission line, centered at around $7 \mathrm{keV}$, as used in the Ginga analysis (Brinkmann et al. 1991) does not describe the shape of this spectral component adequately. From the models in the XS PEC package a broken power law seems to provide a reasonable additional component to the fits. The break energy turns out to be around $7.1 \mathrm{keV}$, the low energy power law rises with energy $\left(\Gamma_{1} \sim-1.0\right)$; the high 
energy part is rather steep $\left(\Gamma_{2} \sim 4.0\right)$, however, the parameters are usually not well determined in the fits. This spectral form is reminiscent of an emission line Compton down-scattered by colder material with moderate $(\tau \sim 5)$ optical depth (Sunyaev $\&$ Titarchuk 1980, Sazonov \& Sunyaev 2000). The number of photons in this scattered component is about 10-15 times higher than the number of photons of the observed iron line, indicating that we are seeing the scattered light from the innermost hot jet, otherwise hidden by the disc material.

However, even with this uncertainty about the exact shape of the additional emission component, the explicit hydrodynamical jet models alone provide sufficient diagnostic power for a relatively reliable determination of the jet parameters.

\subsection{Jet models}

In the "standard" model for the jets the conical outflow of matter cools by adiabatic expansion and emission of thermal radiation. In this model the thermal history of the jet (and its emitted spectrum) is governed by the temperature $T_{0}$ of the gas at the base of the jet, its number density $n_{0}$, its opening angle $\Theta$ and its chemical composition. We adopt a plasma with cosmic abundances (Allen 1973) and assume purely thermal emission from a gas in collisional ionization equilibrium (CIE) at the local temperature. The ionization and recombination rates are taken from Arnaud \& Rothenflug (1983), with corrections for the Fe rates from Arnaud \& Raymond (1992). For the calculation of the optically thin X-ray emission we employed the emission model of Mewe et al. (1985), supplemented by the contribution of relativistic electron-electron bremsstrahlung (Haug 1989) which increases the high energy emission by up to about $10 \%$ at the highest temperatures. In the determination of the line emission we have not included the calculations of the $\mathrm{Fe}$ L-shell transitions (Liedahl et al. 1995), therefore our model will somewhat overestimate the flux near $\sim 1.5 \mathrm{keV}$ and underestimate the flux near $\sim 1 \mathrm{keV}$.

Brinkmann \& Kawai (2000) discussed several variations from this simple approach, including non-uniform density distributions, non-equilibrium ionization of the plasma, and Comptonization of the radiation in the jet. Although the structure of the jets can vary considerably, the changes of the spectral shape of the emitted radiation are perhaps insufficient to provide, for current X-ray instruments, a discriminating diagnostic for the various scenarios.

The flow velocity $v_{\text {jet }}=0.26 c$ is fixed and the opening angle of the jet was confined to be smaller than $\Theta=5^{\circ}$ (Shaham 1981). From recent Chandra observations various values for rather narrow opening angles were determined from the Doppler-broadening of the emission line profiles: 1.22 (Marshall et al. 2002), 2.1 (Namiki et al. (2003), and most recently, 2.7 (Lopez et al. 2003). We will use this last value in our computations and note that the exact value does not significantly affect the form of the emitted X-ray spectrum as discussed by Brinkmann et al. (1988).

The number density $\mathrm{n}_{0}$ of the out-flowing plasma at the bottom of the jet is the primary determining quantity for the length of the X-ray emitting jet due to the linear scaling of the cooling

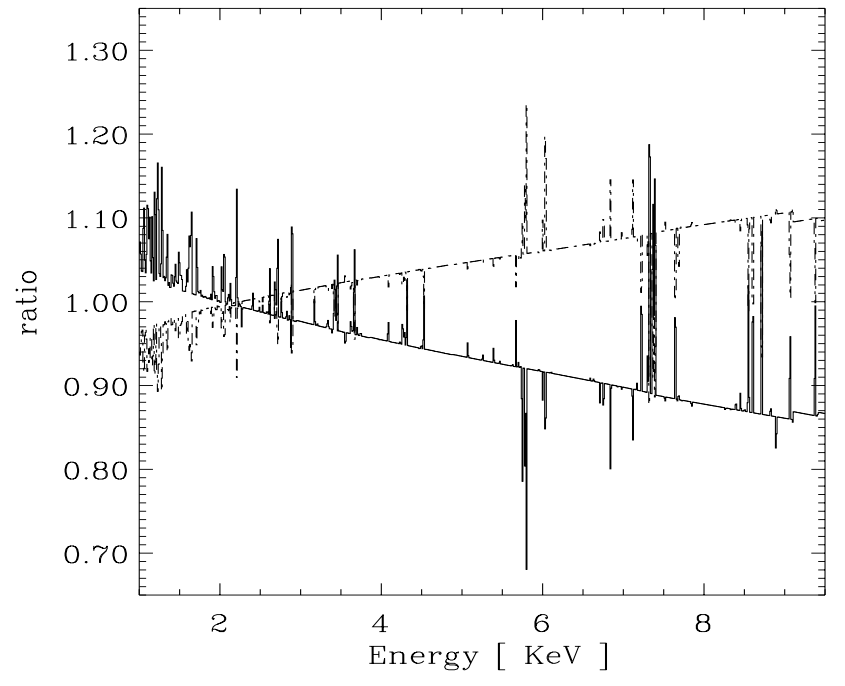

Fig. 5. Ratios of the spectra of standard conical jet models at different temperatures normalized to the model with $k T_{0}=20 \mathrm{keV}$; upper (dash-dotted) curve $k T_{0}=25 \mathrm{keV}$ model, lower curve (full line) $k T_{0}=15 \mathrm{keV}$ model.

time scale with density. The effect of a varying $\mathrm{n}_{0}$ on the shape of the total spectrum is negligibly small, but the visibility and thus the spectral contribution of the red jet to the measured flux depends crucially on the jet length, which is thus the ultimate quantity for the determination of the binary system's parameters.

At a given density changes in the initial temperature $\mathrm{T}_{0}$ lead to relatively small changes of the continuum spectrum but visibly affect the emission line ratios. In Fig. 5 we show the ratio of the spectra of standard conical jet models (blue- and red-shifted contributions) at temperatures of $k T_{0}=25 \mathrm{keV}$ (upper curve) and $k T_{0}=15 \mathrm{keV}$ (lower curve) normalized to the model of a $k T_{0}=20 \mathrm{keV}$ jet. The spectra were appropriately Doppler boosted and the same visibility conditions for the red jet was assumed. The changes in the continuum flux are of the order of $\sim \pm 10 \%$; however, they are found at high photon energies where the signal is low. The biggest changes occur in the line emission regions, which will be used to narrow down the physically acceptable parameter space.

\subsection{Model details}

The adjustment of the jet model parameters to match the line emission in the relatively narrow spectral range of $\sim 5.5-10 \mathrm{keV}$ provides information about the Doppler shifts during the observation, the temperature $T_{0}$ at the base of the jet, the contribution of the red jet, and the (relative) iron and $\mathrm{Ni}$ abundances.

In Fig. 6 we present fits over this high energy band where we have added a broken power law to the jet emission model to account for the deficit of the continuum flux in the iron line region. Clearly visible is the blue-shifted (at $\sim 7.3 \mathrm{keV}$ ) and the red-shifted (at $\sim 5.8 \mathrm{keV}$ ) iron line complex. Note that we will denote here with the term "blue-shift", $1+z_{\mathrm{b}}$, the Doppler shift of the line energies towards higher energies, and vice versa for the "red-shifts", which differs from the commonly used 


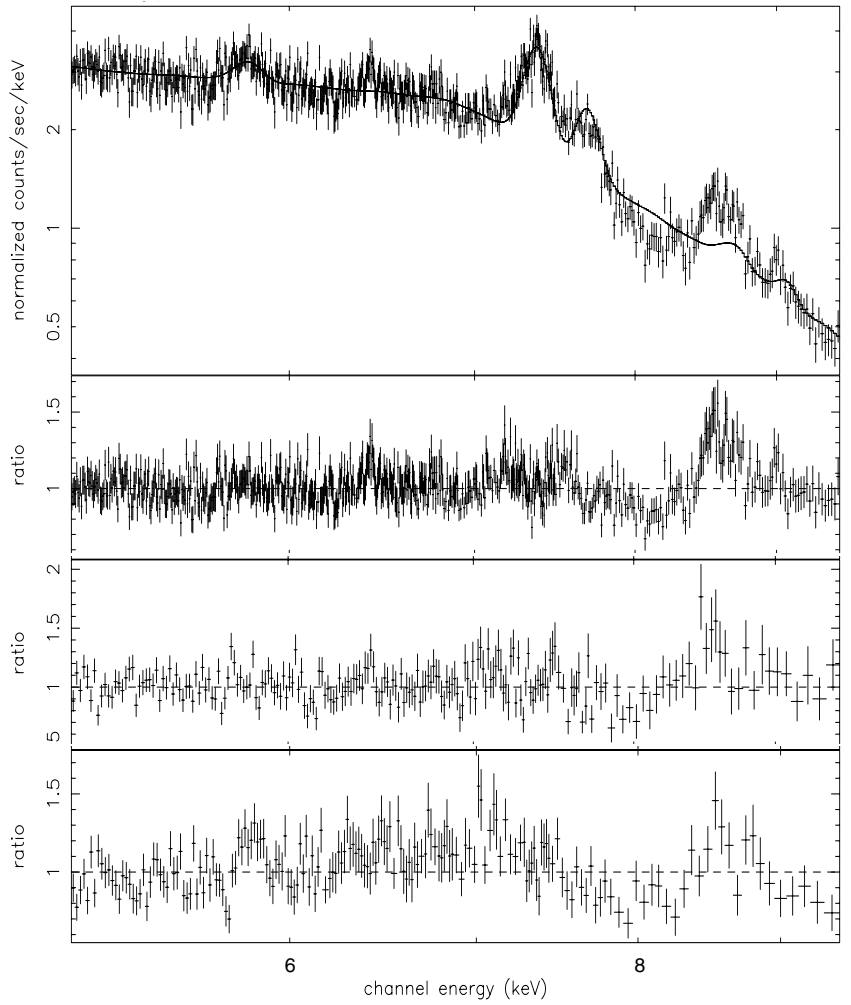

Fig. 6. Fit of a jet model with $k T_{0}=20 \mathrm{keV}$ to the $5.0-9.5 \mathrm{keV}$ energy range. An additional broken power law accounts for the flux deficit in the iron line region (see text). The upper two panels show the PN fit and the model to data ratio; the third panel gives this ratio for the fit to the MOS1 Timing Mode data, the bottom panel that for the MOS2 fit.

definition for the optically shifted lines (in wavelengths) of the "kinematic model" (Margon 1984).

In the calculated model for Fig. 6 we used $1+z_{\mathrm{b}}=1.1046$ and $1+z_{\mathrm{r}}=0.8671 . \Delta z \sim 1 \times 10^{-3}$ seems to be the accuracy in the Doppler shift determination from the PN fits, originating from the statistical quality of the data and changes of the model due to the temperature dependence of the line centroids and the Doppler boosting of the emission. The upper two panels show the PN fit and the model to data ratio for the PN fit; the third panel the model to data ratio for the fit to the MOS1Timing data and in the bottom the corresponding ratio for the MOS2 data. Note that the fit to the MOS1 data yielded an extra Doppler-shift of $\delta z=4.5 \times 10^{-3}$, that to the MOS2 data a $\delta z=2.0 \times 10^{-3}$. However, the data quality is much lower and at high energies the model does not describe the data well and the fits are only marginally acceptable. Quite generally, the overall similarity of the residual structures provides convincing support for the excellent cross calibration of the three instruments.

The ratio between the blue- and the red-shifted Fe XXVI $\mathrm{K}_{\alpha}$ line is a measure of the visibility of the red jet. The above fits indicate that a fraction of about $60 \%$ of the intrinsic iron line emission from the red jet is hidden by the intervening material of the thick disk. The precessional phase expected during this observation is around $\Psi \sim 0.51$ (Eikenberry et al. 2001) or $\Psi \sim 0.544$ (Goranskii et al. 1998), i.e., slightly before the maximal separation of the Doppler curves. At these phases near maximal Doppler shift of the blue jet, the

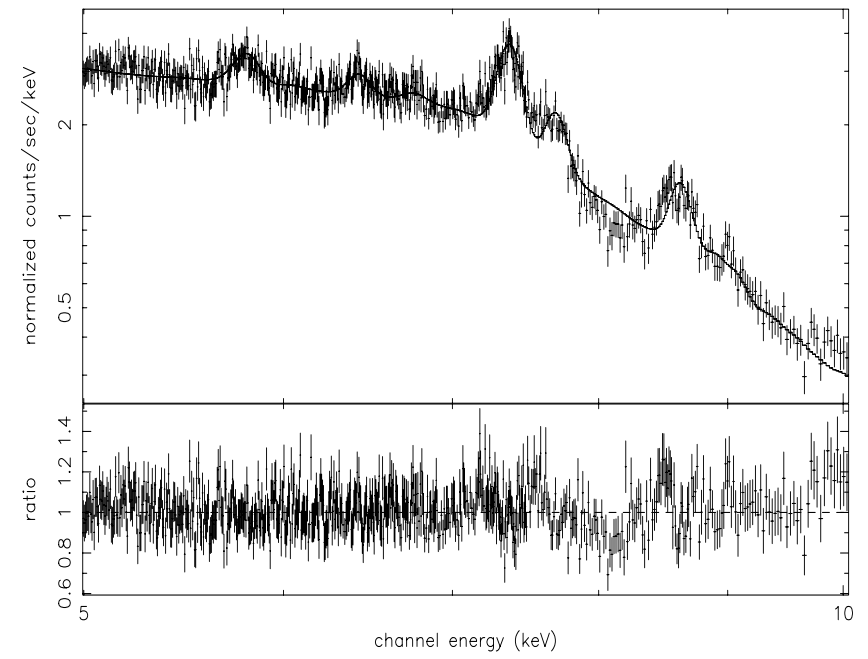

Fig. 7. $\mathrm{PN}$ fit to a $k T_{0} \sim 16 \mathrm{keV}$ model in the $5.0-10 \mathrm{keV}$ energy range. The $\mathrm{Ni}$ abundance of the models was increased to eight times the solar value and a narrow neutral iron line was added.

contribution of the red jet is small and hard to estimate. While the position of the red-shifted iron line coincides with the predictions of the Doppler shifts, the blue shifted line seems to require later phases.

Increasing the temperature of the model to $k T_{0}=25 \mathrm{keV}$ (or decreasing it to $k T_{0}=15 \mathrm{keV}$ ) yields iron line ratios which appear to be less in agreement with the data. In Fig. 7 we show the fit with a table model to the data. (This user-defined model contains a grid of numerically determined spectra with the base temperature of the jet as a fit parameter). The Nickel abundance in the model has been increased to eight times the solar value. The fitted temperature is $k T_{0}=16.2 \pm 0.85 \mathrm{keV}$. The fit is not good but acceptable with $\chi_{\text {red }}^{2}=1.22 / 619$ d.o.f. Obviously, we poorly estimated the contribution of the redshifted jet, which becomes apparent in the structure of the residuals at $\sim 5.8 \mathrm{keV}$ and $\gtrsim 7 \mathrm{keV}$. The data further suggest the presence of a neutral iron line and the inclusion of an extra narrow line at $E_{1}=6.40 \pm 0.03 \mathrm{keV}$ with a normalization of $n_{1}=(8.1 \pm 0.03) \times 10^{-5}$ photons $/ \mathrm{cm}^{2} / \mathrm{s}$ improved the fit significantly. Applying the same model to the MOS1 data results in a temperature of $k T_{0}=18.52 \pm 2.25 \mathrm{keV}$, with iron line parameters very similar to the $\mathrm{PN}$ results at a $\chi_{\text {red }}^{2}=1.32 / 207$ d.o.f.

The Ni abundance had to be increased considerably, as already indicated by the ASCA data (Kotani et al. 1997) but still seems not quite to match the observed flux. Due to the dominance of the iron emission lines the iron abundance of the jets' plasma plays an important role in the spectral analysis. Decreasing the iron abundance to $50 \%$ of the solar value requires the normalization of the model to be increased, which, in turn, leads to an excess of model flux at lower energies. Adjusting the model flux at low energies and providing the missing hard flux with the scattering component yields a large mismatch in the continuum at around $8 \mathrm{keV}$, considerably larger than seen in Fig. 7, and to systematic differences in the line fluxes at low energies. On the other hand, an increase of the iron abundance to twice the cosmic value leads to a strong reduction (by a factor of 2) of the model flux in the high energy 
fit and, correspondingly, to a large flux deficit at lower energies. Further, the low energy line emission does not match the measurements. Thus the XMM spectra seem to favor iron abundances near the cosmic value, but highly increased $\mathrm{Ni}$ abundances.

Quite generally, due to the limited photon statistics and the only poorly known shape of the scattering component at $\sim 7 \mathrm{keV}$ we find in the fits a strong degeneracy between the contribution of the red jet, the chemical abundances of the jet material (in particular of $\mathrm{Fe}$ and $\mathrm{Ni}$ ), and the initial temperature of the jet at its base. In Table 2 we summarize the fitted parameters for all four orbits, assuming a Ni overabundance by a factor of eight, and S and Si over-abundances by a factor of two compared to the cosmic values (see below). The spectral contribution of the red jet is parameterized by a characteristic temperature along the jet from which the emission is visible. In contrast to the actual physical length, which is strongly dependent on the initial plasma density of the jet, the temperature provides, to first order, a scale-free measure of the red jet's contribution. The listed temperatures in Table 2 are obtained from the high energy fits $(5-10 \mathrm{keV})$, the absorption from the low energy fits (0.8-3.3 keV; see Sect. 3.4). In the last column $\eta$ gives (in percent) the contribution of the flux from the scattering component compared to the total $0.5-10 \mathrm{keV}$ flux from the thermally radiating jets.

\subsection{The low energy spectrum}

An extrapolation of the above model to the whole $0.7-10 \mathrm{keV}$ energy band and leaving the absorption as an additional free parameter results in both cases in worse fits: for the PN we obtain an absorption column density of $N_{\mathrm{H}}=(9.46 \pm 0.08) \times 10^{21} \mathrm{~cm}^{-2}$, a temperature of $k T_{0}=17.7 \pm 0.97 \mathrm{keV}$, and a $\chi_{\text {red }}^{2}=1.42 / 1418$ d.o.f. The MOS1 fits resulted in $N_{\mathrm{H}}=(1.03 \pm 0.1) \times 10^{22} \mathrm{~cm}^{-2}$, a temperature of $k T_{0} \gtrsim 25 \mathrm{keV}$ and a $\chi_{\text {red }}^{2}=1.94 / 494$ d.o.f. In all cases these total fits result in characteristic residuals at low energies and in the iron line region and different temperatures of the fitted models.

As at low energies the MOS is more sensitive to spectral features we can clearly identify some deficits in the model flux near energies where the emission lines from the red-shifted jet are expected. This suggests that in the models used above, the contribution of the red jet was underestimated.

In Fig. 8 we show the contributions of the two jets to the total emission for a typical model used in the above fits. The emission from the red-shifted jet contributes noticeably only at $\sim 5.8 \mathrm{keV}$, at $\sim 7 \mathrm{keV}$ and at energies below $3 \mathrm{keV}$. At unfavorable red-/blue-shifts some prominent emission lines from the red jet can coincide with the lines from the blue jet, which then leads to the previously claimed 'invisibility' of the red jet (Watson et al. 1986) or extra absorption in the system (Kotani 1996). It is therefore tempting to try to adjust the contribution of the red jet by changing the fraction of obscuring of the inner parts of the red jet. However this concept does not yield the desired result as it mainly affects the red-shifted high energy Fe lines. For example, if we vary the obscuration of the inner red jet such that its total photon flux changes by $\sim 10 \%$ the flux at

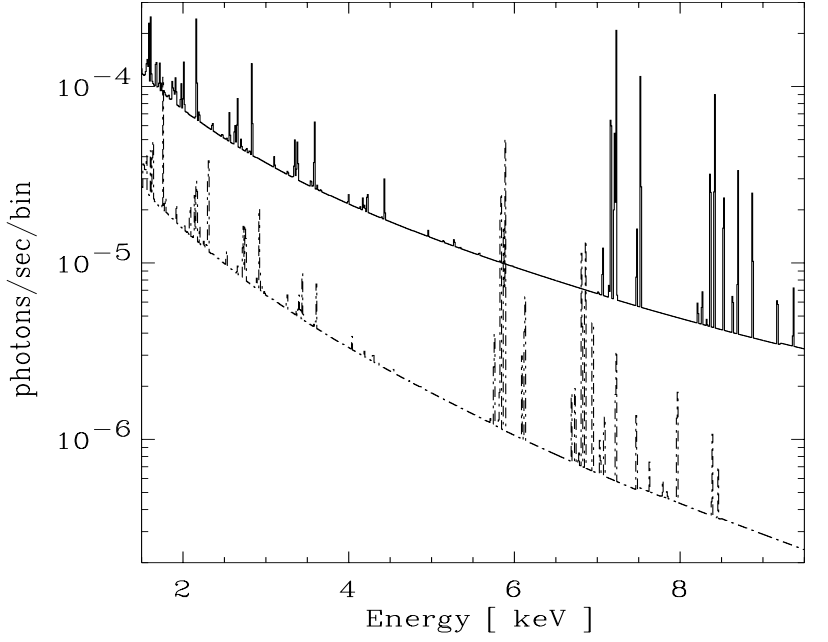

Fig. 8. Spectra of a blue- and red-shifted jet model with $k T_{0}=$ $17.5 \mathrm{keV}$ in the $1.5-9.5 \mathrm{keV}$ energy range.

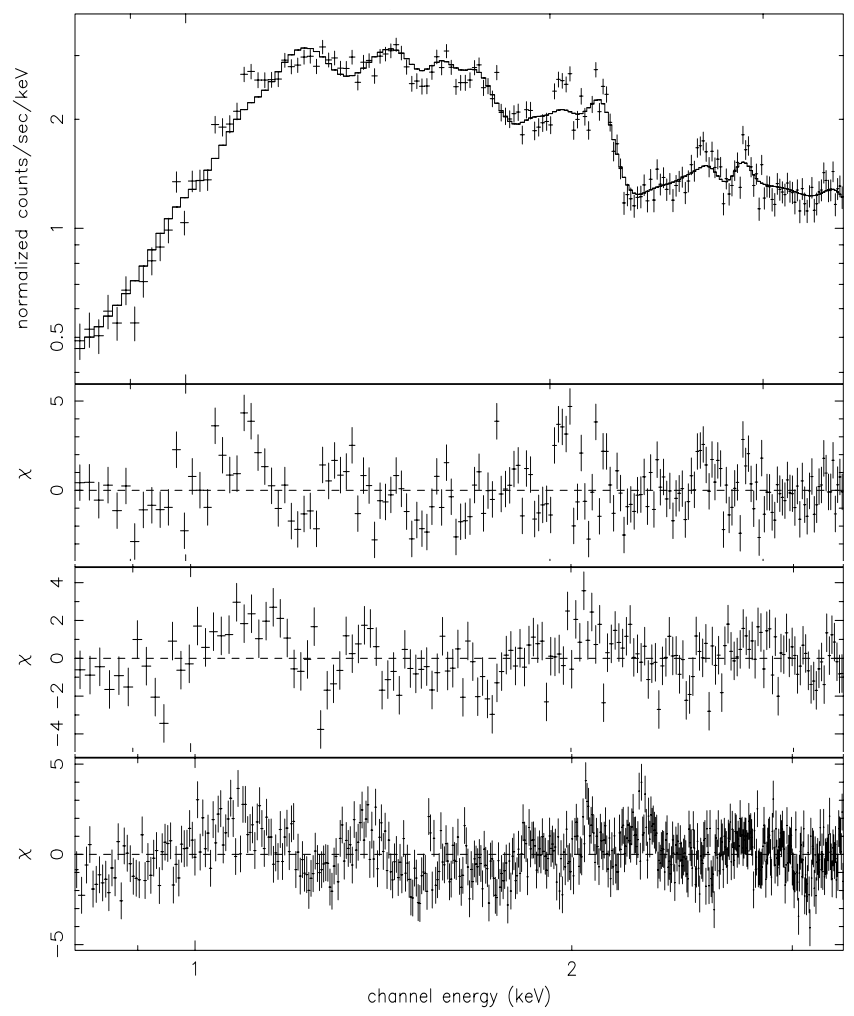

Fig. 9. Fit of a $k T_{0}=17 \mathrm{keV}$ model to the soft $0.8-3.3 \mathrm{keV}$ data of the MOS1 data and the residuals of the fits to the MOS1, MOS2 and PN data.

the $\mathrm{Si} / \mathrm{S}$ line energies changes by $\lesssim 4 \%$ whereas the flux at the red-shifted high energy Fe lines $(\sim 5.8 \mathrm{keV})$ changes by $40 \%-$ with dramatic effects on the high energy fits.

This means that possible modifications of the contribution from the red jet are limited and that systematic residuals remain at low energies. The upper panels in Fig. 9 show a model fit to the MOS1 data in the $0.8-3.3 \mathrm{keV}$ energy range and the resulting residuals. The corresponding residuals from the MOS2 fit are given in the third panel, in the bottom panel those of the PN fit. In all three detectors we find similar characteristic 
Table 2. Results of the EPIC fits.

\begin{tabular}{|c|c|c|c|c|c|c|c|c|c|}
\hline Orbit & $\begin{array}{c}\text { Precession phase } \\
\Psi \ddagger\end{array}$ & $\begin{array}{c}\text { Binary phase } \\
\Phi^{\dagger}\end{array}$ & Instrument & $\begin{array}{c}k T_{0} \\
{[\mathrm{keV}]}\end{array}$ & $\begin{array}{l}k T_{\text {red }} \\
{[\mathrm{keV}]}\end{array}$ & $1+z_{b}$ & $1+z_{\mathrm{r}}$ & $\begin{array}{c}N_{\mathrm{H}} \\
{\left[\times 10^{22} \mathrm{~cm}^{-2}\right]}\end{array}$ & $\begin{array}{c}\eta \\
{[\%]^{*}}\end{array}$ \\
\hline 607 & 0.51 & 0.045 & $\begin{array}{c}\text { PN } \\
\text { MOS1 } \\
\text { MOS2 }\end{array}$ & $13.21 \pm 0.74$ & $7.0 \pm 1.0$ & 1.0806 & 0.8799 & $0.86 \pm 0.02$ & 1.2 \\
\hline 608 & 0.52 & 0.193 & $\begin{array}{c}\text { PN } \\
\text { MOS1 } \\
\text { MOS2 }\end{array}$ & $\begin{array}{l}13.67 \pm 0.74 \\
17.55 \pm 3.45 \\
13.45 \pm 2.38\end{array}$ & $5.8 \pm 1.0$ & 1.0728 & 0.8783 & $\begin{array}{l}0.89 \pm 0.01 \\
0.99 \pm 0.03 \\
0.95 \pm 0.05\end{array}$ & $\begin{array}{l}1.6 \\
2.2 \\
0.9\end{array}$ \\
\hline 609 & 0.53 & 0.344 & $\begin{array}{c}\text { PN } \\
\text { MOS1 } \\
\text { MOS2 }\end{array}$ & $\begin{array}{l}14.94 \pm 1.05 \\
13.44 \pm 2.16 \\
13.78 \pm 2.66\end{array}$ & $6.4 \pm 1.0$ & 1.1028 & 0.8613 & $\begin{array}{l}0.91 \pm 0.03 \\
0.98 \pm 0.02 \\
0.96 \pm 0.02\end{array}$ & $\begin{array}{l}1.6 \\
9.3 \\
1.7\end{array}$ \\
\hline 610 & 0.54 & 0.497 & $\begin{array}{c}\text { PN } \\
\text { MOS1 } \\
\text { MOS2 }\end{array}$ & $\begin{array}{l}16.01 \pm 0.92 \\
13.99 \pm 1.67 \\
15.54 \pm 2.21\end{array}$ & $6.0 \pm 1.0$ & 1.1046 & 0.8671 & $\begin{array}{l}0.91 \pm 0.02 \\
0.98 \pm 0.02 \\
1.03 \pm 0.02\end{array}$ & $\begin{array}{l}2.1 \\
1.6 \\
1.5\end{array}$ \\
\hline
\end{tabular}

$\dagger$ Phases from Goranskii et al. (1998).

* Phase 0 as defined by Margon (1984); add 0.342 for the Goranskii et al. values.

* Fraction of flux in the scattering component to the total $0.5-10 \mathrm{keV}$ flux (in percent).

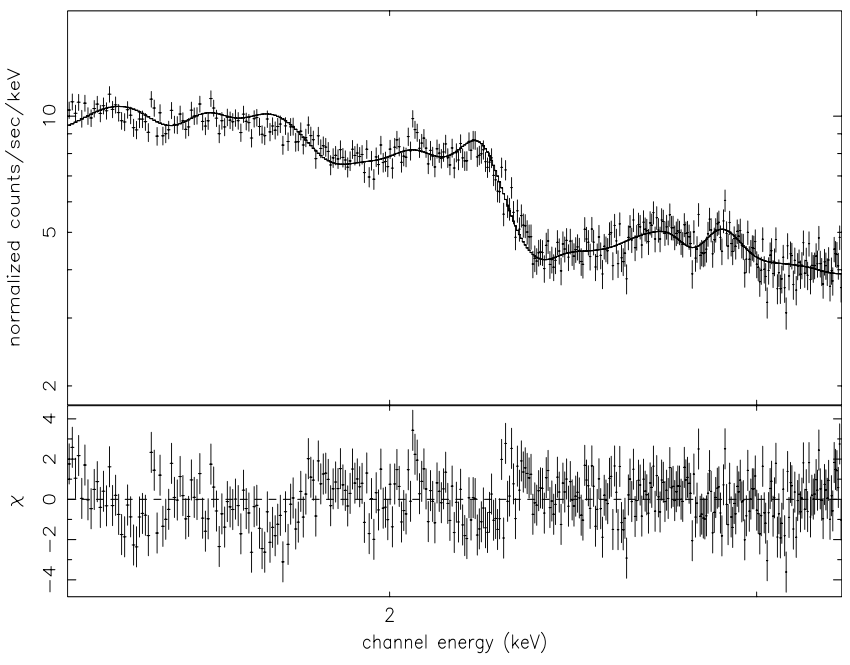

Fig. 10. Blowup of the $1.5-3.3 \mathrm{keV}$ energy region for the $\mathrm{PN}$ fit with $\mathrm{Si}$ and $\mathrm{S}$ abundances two times the cosmic values.

residuals: in the energy region of $\sim 1.05-1.1 \mathrm{keV}$ the model under-estimates the data. The deficit must be attributed to the incorrect treatment of blue-shifted Fe-L emission at $\lesssim 1 \mathrm{keV}$ in Mewe's original emission model. The deficit in the model around $1.35-1.4 \mathrm{keV}$ might have similar causes. However, an incorrect determination of the contribution of the red-shifted emission or different $\mathrm{Fe} / \mathrm{Ni}$ abundances could be responsible as well.

At $\gtrsim 2 \mathrm{keV}$ and $\sim 2.2 \mathrm{keV}$ we find excess flux from blueshifted SiXIII - SiXIV emission; the two residuals around $2.7 \mathrm{keV}$ and $2.9 \mathrm{keV}$ can be attributed to SXV - SXVI lines.

An increase of the abundances of $\mathrm{Si}$ and $\mathrm{S}$, both by a factor of $\sim 2$ improves the fits considerably, which is demonstrated in Fig. 10, showing the relevant energy band (1.5-3.3 keV) from the PN fits. A more accurate determination of the abundances is inhibited by the systematic differences between the three detectors; however, over-abundances by factors of 3 seem to be ruled out.

These low energy fits provide perhaps the best determination of the absorption towards the source: the PN fit $\left(\chi_{\text {red }}^{2}=\right.$ $1.42 / 492$ d.o.f.) gives $N_{\mathrm{H}}=(9.07 \pm 0.02) \times 10^{21} \mathrm{~cm}^{-2}$, the MOS1 fit $\left(\chi_{\text {red }}^{2}=2.22 / 160\right.$ d.o.f. $)$ gives $N_{\mathrm{H}}=(9.83 \pm 0.02) \times$ $10^{21} \mathrm{~cm}^{-2}$, and the MOS2 fit $\left(\chi_{\text {red }}^{2}=1.66 / 161\right.$ d.o.f. $)$ gives $N_{\mathrm{H}}=(1.03 \pm 0.02) \times 10^{22} \mathrm{~cm}^{-2}($ see Table 2$)$.

\subsection{Orbit 607}

The observation in orbit 607 took place during the eclipse of the central parts of the jets by the primary star, thus we expect a softer spectrum and less confusion from the scattering region on the disc. The possible obscuration of the red jet by the primary star is, however, another unknown factor for the model adjustment.

Fitting the emission model to the PN data (no scientifically usable data are available from the MOS cameras) yields a temperature of $k T_{0}=13.21 \pm 0.74 \mathrm{keV}$ with a $\chi_{\text {red }}^{2}=1.167 / 779$ d.o.f. for the high energy data. Nearly the same $\chi_{\text {red }}^{2}$ could be obtained by fitting the whole energy range $(0.8-10 \mathrm{keV})$ with a slightly higher temperature model $\left(k T_{0}=17.49 \pm 1.75 \mathrm{keV}\right)$, which demonstrates the relatively large insensitivity of the fitted parameters of the model due to the lower statistical quality of these data. Correspondingly, the contribution of the red jet remains rather uncertain. Notice that the relative contribution of the scattering component to the total emission is reduced, which is expected when the inner parts of the disc are obscured by the primary. 


\subsection{Orbit 608}

During the orbit 608 observation the source was out of eclipse, at a binary phase at which the light curves of previous Ginga and ASCA observations showed considerable scatter. Further, the background during the observation was quite noisy, leading to a reduced observing efficiency, especially for the MOS cameras. The fit to the high energy PN data yielded a temperature of $k T_{0}=13.67 \pm 0.74 \mathrm{keV}$ with a $\chi_{\text {red }}^{2}=1.297 / 513$ d.o.f.; for the total band the values were slightly higher and the quality of the fit was low $\left(k T_{0}=14.32 \pm 0.95 \mathrm{keV}, \chi_{\text {red }}^{2}=1.719 / 1361\right.$ d.o.f.). The MOS fits resulted in similar temperatures with considerably better $\chi_{\text {red }}^{2}$, possibly due to the lower quality of the data. Interestingly, the fitted Doppler shift of the blue jet was smaller than those obtained for the PN and MOS2 detectors by $\delta(1+z) \sim-7.5 \times 10^{-3}$ and clearly incompatible with fitted PN value.

\subsection{Orbit 609}

In this orbit we have the shortest observation, characterized by very high background activity. The spectra are very noisy and of low signal to noise ratio and, correspondingly, the errors of the fit parameters are rather large. The obtained red shifts deviate markedly from the expected Doppler curve and the extreme value of the calculated flux fraction in the scattered component for the timing mode data clearly demonstrate the limited data quality.

\section{Discussion}

The analysis of the EPIC data have confirmed the model of thermally radiating jets for the X-ray emission of SS 433. Due to the limited photon statistics and the existence of the scattering component peaking at energies around 6-7 keV, an exact determination of the temperature at the base of the visible jet was not possible, but the best estimates are around $k T_{0} \sim 17 \pm 2 \mathrm{keV}$. A determination of the plasma temperature from the FeXXV/FeXXVI line ratio is limited by the unknown shape of the scattering component. PN and MOS fits show in general good agreement with each other, but with specific differences in the fitted temperatures $\left(k T_{\mathrm{Mos}} \leq k T_{\mathrm{pn}}\right)$. The positions of the fitted iron line energies differ only by $\sim 20 \mathrm{eV}$ in the three detectors.

The red jet contributes about $30-40 \%$ to the total photon flux, but the exact value is not well constrained by the current data. Most of the emission occurs at low energies where the EPIC instruments have lower energy resolution, making a separation of the red and blue shifted lines problematic; at high energies the contribution of the red-shifted Fe-line is contaminated by the scattering component.

This scattering component was not detected in the EXOSAT or ASCA data but was seen in the Ginga data due to the LAC's large effective area. The limited spectral resolution of the instrument allowed the modeling of this component with a broad, stationary Gaussian line. The EPIC data show a more complicated shape. From the "canned" models in the XS PEC package a broken power law provided the best representation of the data. The errors of the slopes of the fitted parameters are quite large, but in all fits they are consistently around $\Gamma_{\text {soft }} \sim-1, \Gamma_{\text {hard }} \sim 3-4$, with a break energy $E_{\text {break }} \sim 7.1 \mathrm{keV}$. The total flux in this component is only of the order of $1-2 \%$ of the thermal emission of the jet and seems to correlate with the viewing conditions onto the central parts of the disk. The spectral shape and the fitted parameters are reminiscent of the Comptonized flux from an iron line in a sphere of gas with temperature $k T_{\mathrm{e}} \sim 0.1 \times h v$ and an optical depth of $\tau \sim 7$ (c.f. Fig. 4 of Sunyaev \& Titarchuk 1980).

The typical residuals of the fits indicate, however, a more complex shape. In particular, the hard power law component over-predicts the count rate around $8 \mathrm{keV}$ in all fits. This uncertain shape effects the accuracy of any determination of the jet parameters from the high energy fits, especially as the jet temperature, the ratios of the iron emission lines, the elemental abundances and the redshift are strongly inter-connected.

The outflowing gas shows over-abundances in different elements: $\mathrm{Si}$ and $\mathrm{S}$ by factors of $\sim 2, \mathrm{Ni}$ by $\sim 8$. A high overabundance of Fe seems unlikely but definite values can only be attained from a better modeling of the scattering component. This overabundance of heavy elements might be a tracer for the past explosion of the massive progenitor of the compact star; elemental synthesis of for example $\mathrm{Ni}$ in the inner parts of the accretion disk appears unlikely due to the extremely high temperatures required - although the very high temperatures of the observable jet flow, indicating even more extreme conditions at the base of the jets, remain so far to be explained as well.

At the high temperatures envisaged electron - electron bremsstrahlung should contribute to the emission; however, even at higher energies this is only a 6-10\% effect.

The fitted shapes of the $\mathrm{Fe}$ and $\mathrm{Ni}$ lines indicate some systematic residuals; however, differences between the two MOS cameras and the PN make it hard to distinguish statistical variations from genuine physical effects. Further, if we use for the PN fits only single events these deviations are strongly reduced which indicates that the fits are affected by an unusually high fraction of double events due to the high background level. On the other hand, fits with single events are of only limited statistical significance.

The obtained red / blueshifts appear, at first glance, to deviate quite a bit from the "classical" precessional Doppler curve (Margon 1984). However, for the higher accuracy achieved in the Doppler shift determination the nodding motion of the disk (Katz et al. 1982) cannot be ignored, as already shown in recent optical observations (Gies et al. 2002a). In Fig. 11 we plot the red/blue shifts around the times of our observations (note that we use here the "conventional" shifts in wavelength space). The ephemeris of the nodding motion is taken from Goranskii et al. (1998); for the semi-amplitude we used a value of $\delta z=0.075$ in accordance with previous investigations (Katz et al. 1982, Gies et al. 2002a). The size of the symbols represents the systematic errors from the fits to the different EPIC instruments. Inside their errors the data are consistent with this Doppler curve (apart from the observation in orbit 609). The uncertainties of the ephemeris do not allow a determination of light travel effects from the X-ray data alone. 


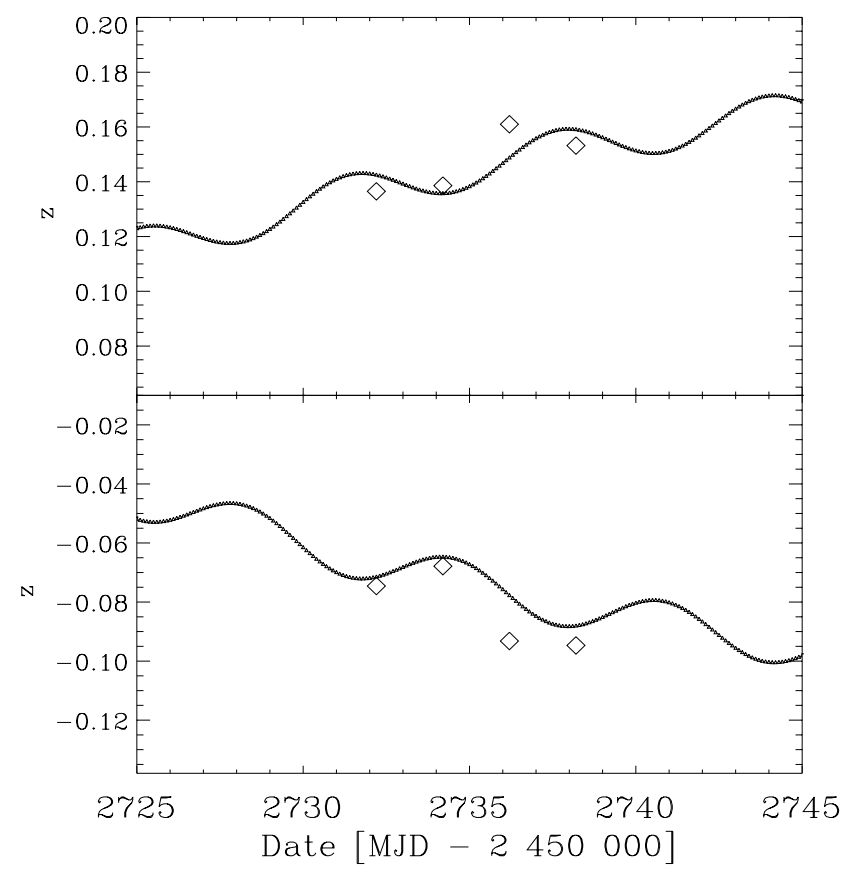

Fig. 11. Radial velocity curve according to the ephemeris of Goranskii et al. 1998; including nodding motions (Katz et al. 1982). The symbol sizes correspond to the systematic uncertainties of the redshift determination from the fits.

The fits at lower energies provide the currently best estimate of neutral absorption towards the source of $N_{\mathrm{H}}=$ $\sim 9 \times 10^{21} \mathrm{~cm}^{-2}$ which is higher than the column density obtained for the lobes of W50 (Brinkmann et al. 1996) and indicates the presence of extra material around SS 433. However, the complex emission below $\sim 2 \mathrm{keV}$ still needs some model refinement.

\section{Conclusions}

The XMM-Newton observations of SS 433 have confirmed the general picture that the X-ray emission of the source is the thermal emission from the two relativistic jets.

The determining physical parameter of the jet emission, the density $n_{0}$ at the base of the jets, cannot be obtained from the spectral analysis. The fact that during the four observations the soft flux remains nearly constant and that only the hard emission from the inner parts of the jets varies indicates that the jet dimensions must exceed the typical length scales of the binary system, i.e. $\sim 2 \times 10^{12} \mathrm{~cm}$ (Gies et al. 2002b).

Taking the masses of the two stars of $M_{0}=19 M_{\odot}$ and $M_{\mathrm{x}}=11 M_{\odot}$, as given by Gies et al. (2002b) and assuming that the primary fills its Roche lobe, we find that during eclipse the innermost $\sim 1.2 \times 10^{12} \mathrm{~cm}$ of the blue jet are hidden by the star. Matching the measured intensities in and out of eclipse this would imply that the X-ray jet is rather long $\left(\sim 4 \times 10^{13} \mathrm{~cm}\right)$ and that the densities at the base of the jets must be $n_{0} \sim 10^{12} \mathrm{~cm}^{-3}$. The total kinetic energy of the outflowing matter is then $\sim 5 \times$ $10^{39}$ erg s $^{-1}$.

These values are rather extreme and it should be noticed that the mass ratio of $q=0.57$, obtained for the above masses, is incompatible with the values of $q \sim 0.15$, deduced from the Ginga X-ray light curves (Brinkmann et al. 1989) and $q \sim 0.22$, found from ASCA observations (Kotani 1997). A more detailed intensity and spectral analysis of the eclipse process will certainly clarify these discrepancies.

We find non-cosmic abundances for some of the prominent $\mathrm{X}$-ray line emitting elements: $\mathrm{Ni}$ is over-abundant by a factor of $\sim 8, \mathrm{~S}$ and $\mathrm{Si}$ by a factor of $\sim 2, \mathrm{Fe}$ seems to be close to the cosmic value. However, more accurate values cannot be given due to the, in this respect, limited quality of the data.

The fits are strongly affected by the unknown shape of a scattering component around $7 \mathrm{keV}$, which was already found in the Ginga data ("stationary line") but not in later ASCA and Chandra observations. This component and the presence of a Fe fluorescent line at $\sim 6.4 \mathrm{keV}$, with a typical photon flux of $\sim$ few $\times 10^{-5}$ photons $/ \mathrm{cm}^{2} / \mathrm{s}$, are strong indicators of Compton scattering of the emission from the base of the jets from the surrounding cold disk/wind material. The presence of this component during eclipse, even at reduced intensity, argues for a relatively large scattering region and/or an extended disk. The strength of the neutral iron line can further provide important information about the amount of scattering in the system.

We do not find statistically significant flux variability on short time scales; the noisiness of the light curves can be directly related to flaring activity of the background.

The accuracy of the spectral modeling at high energies is limited by the presence of the scattering component and its complex interplay with the ratio of the $\mathrm{Fe} / \mathrm{Ni}$ abundances; at low energies the spectral resolution of the detectors, the uncertain contribution of the red jet to the emission, and the statistical quality of the data are limiting factors. On the other hand the data seem to require a modeling of the emission with the most accurate atomic emission codes as well as the consideration of more accurate hydrodynamical models, compared to the "canonical jet" model used here.

As the object is heavily absorbed at low energies the RGS data are only of moderate quality. However, the detection of at least some prominent emission lines with the gratings might provide additional constraints on the physical and chemical parameters of the jets. Their analysis will be presented in a forthcoming paper.

In principle current X-ray missions like XMM-Newton and Chandra provide the sensitivity and spectral resolution to disentangle details in the X-ray emission from the SS 433 jets which were previously not possible. However, we found that even then the exact determination of single specific parameters of the X-ray emission, for example the Fe-line fluxes, are insufficient for an unambiguous determination of the jet's parameters; a full range of observationally accessible data and detailed modeling is required to understand this enigmatic object.

Acknowledgements. This work is based on observations with XMMNewton, an ESA science mission with instruments and contributions directly funded by ESA Member States and the USA (NASA). We are very grateful to E. Haug for supplying a Fortran program for the calculation of the relativistic $\mathrm{e}^{-}-\mathrm{e}^{-}-$bremsstrahlung and M. Bauer for his calculations of the binary geometry. WB thanks the JSPS for financial support and TITECH for hospitality where part of the research was done. 


\section{References}

Allen C. W. 1973, Astrophysical Quantities, 3rd ed. (London: Athlone Press)

Arnaud M., \& Rothenflug R. 1985, A\&AS, 60, 425

Arnaud M., \& Raymond J. 1992, ApJ, 398, 394

Brinkmann W. 1992, A\&A, 254, 460

Brinkmann, W., Fink, H. H., Massaglia, S., Bodo, G., \& Ferrari, A. 1988, A\&A, 196, 313

Brinkmann W., Fink H. H., Smith A., \& Haberl F. 1989b, A\&A, 221, 385

Brinkmann W., Kawai N., \& Matsuoka M. 1989, A\&A, 218, L13

Brinkmann W., Kawai N., Matsuoka M., \& Fink H. H. 1991, A\&A, 241,112

Brinkmann W., Aschenbach B., \& Kawai N. 1996, A\&A, 312, 306

Brinkmann, W., \& Kawai, N. 2000, A\&A, 363, 640

Ehle, M., Breitfellner, M., Dahlem, et al. 2001, XMM-Newton Users' Handbook http://xmm.vilspa.esa.es/xmm_user_support/ external/documentation/uhb_frame.shtml

Eikenberry, S. S., Cameron, P. B., Fierce, B. W., et al. 2001, ApJ, 561, 1027

Gies, D. R., McSwain, M. V., Riddle, R. L., et al. 2002a, ApJ, 566, 1069

Gies, D. R., Huang, W., \& McSwain, M. V. 2002b, ApJ, 578, L67

Goranskii, V. P., Esipov, V. F., \& Cherepashchuk, A. M. 1998, Astron. Rep., 42, 209

Grindlay, J. E., Band, D., Seward, F., et al. 1984, ApJ, 277, 286

Haug, E. 1989, A\&A, 218, 330

Hillwig, T. C., Gies, D. R., Huang, W., et al. 2004, [arXiv:astro-ph/0403634]

Katz, J. I., Anderson, S. F., Margon, B., \& Grandi, S. A. 1982, ApJ, 260,780

Kawai N., Matsuoka M., Pan H. C., \& Stewart, G. C. 1989, PASP, 41, 491

Kemp, J. C., Henson, G. D., Kraus, D. J., et al. 1986, ApJ, 305, 805

Kotani, T. 1997, Ph.D. Thesis, University of Tokyo
Kotani T., Kawai N., Matsuoka M., \& Brinkmann W. 1996, PASJ, 48, 619

Kotani T., Kawai N., Matsuoka M., \& Brinkmann W. 1997, IAU Coll. 163, ed. D. T. Wickramasinghe, L. Ferrario, \& G. V. Bicknell, 370

Kotani, T., Trushkin, S., \& Denissyuk, E. K. 2003, in New Views on Microquasars, ed. P. Durouchoux, Y. Fuchs \& J. Rodriguez, Centre for Space Physics, India, Kolkata, 265

Liedahl, D. A., Osterheld, A. L., \& Goldstein, W. H. 1995, ApJ, 438, L115

Lopez, L. A., Marshall, H. L., Kane, J. F., Schulz, N. S., \& Canizares, C.R. 2003, BAAS, 203, 31.06

Margon, B. 1984, ARA\&A, 22, 507

Margon, B., \& Anderson, S. F. 1989, ApJ, 347, 448

Marshall, F. E., Swank, J. H., Boldt, E. A., Holt, S. S., \& Serlemitsos, P.J. 1979, ApJ, 230, L145

Marshall, H. L., Canizares, C. R., \& Schulz, N. S. 2002, ApJ, 564, 941

Mewe R., Gronenschild E. H. B. M., \& Van den Oord G. H. J. 1985, A\&AS, 62, 197

Migliari, S., Fender, R., \& Mendez, M. 2002, Science, 297, 1673

Mirabel, I. F., \& Rodriguez, L.F. 1998, Nature 392, 673

Namiki, M., Kawai, N., Kotani, T., \& Makishima, K. 2003, PASJ, 55, 281

Ricketts, M. J., Hall, D., Page, C. G., Pounds, K.A., \& Sims, M. R. 1981, Vistas Astron., 25, 71

Sazonov, S. Y. \& Sunyaev, R. A. 2000, ApJ, 543, 28

Seward, F. D., Page, C. G., Turner, M. J., \& Pounds, K. A. 1976, MNRAS, 175, 39

Shaham J. 1981, Vistas Astron., 25, 217

Stewart, G. C., Watson, M. G., Matsuoka, M., et al. 1987, MNRAS, 228, 293

Sunyaev, R. A. \& Titarchuk, L. G., A\&A, 86, 121

Titarchuk L. 1994, ApJ, 434, 313

Watson, M. G., Stewart, G. C., Brinkmann, W., \& King, A. R. 1986, MNRAS, 222, 261

Yamauchi, S., Kawai, N., \& Aoki, T. 1994, PASJ, 46, L109

Yuan, W., Kawai, N., Brinkmann, W., \& Matsuoka, M. 1995, A\&A, 297,451 